\title{
The deep vein thrombosis caused by lymphocele after endoscopic extraperitoneal radical prostatectomy and pelvic lymph node dissection
}

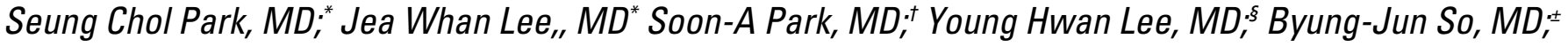 \\ Joung Sik Rim, $M D^{*}$
}

*Department of Urology, Wonkwang University School of Medicine and Hospital, Institute of Wonkwang Medical Science, Iksan, Korea; 'Department of Nuclear Medicine, Wonkwang University School of Medicine and Hospital, Institute of Wonkwang Medical Science, Iksan, Korea; ¿ ¿ Department of Radiology, Wonkwang University School of Medicine and Hospital, Institute of Wonkwang Medical Science, lksan, Korea; 'Department of Surgery, Wonkwang University School of Medicine and Hospital, Institute of Wonkwang Medical Science, Iksan, Korea

Cite as: Can Urol Assoc J 2011;5(3):e40-e43; D01:10.5489/cuaj.10093

\section{Abstract}

Pelvic lymphocele is a postoperative complications than can result after endoscopic extraperitoneal radical prostatectomy and pelvic lymph node dissection. Radical prostatectomy have many risk factors of deep vein thrombosis including location of target organ, malignancy, old age, Trendelenburg position, pelvic lymph node dissection, and long procedure time. A 57-year-old man with a localized prostate cancer was treated with endoscopic extraperitoneal radical prostatectomy and pelvic lymph node dissection. Deep vein thrombosis was detected as a first sign of pelvic lymphocele. Lymphocele was managed with a percutaneous drainage without sclerosant. We report a case of deep vein thrombosis due to pelvic lymphocele after endoscopic extraperitoneal radical prostatectomy.

\section{Introduction}

Laparoscopic transperitoneal and endoscopic extraperitoneal radical prostatectomy are established techniques for the management of localized prostate cancer in numerous urologic centers worldwide. Complications of endoscopic extraperitoneal radical prostatectomy, according to Clavien system, are $0.45 \%$ intraoperatively, $8.6 \%$ in the early postoperative period and $0.3 \%$ in the late postoperative period. ${ }^{1}$ Pelvic lymphocele is a postoperative complication that can result after pelvic lymphadenectomy for the treatment of pelvic malignancy. There is a high incidence $(30 \%)$ of subclinical lymphoceles after laparoscopic pelvic lymphadenectomy for prostatic cancer. ${ }^{2}$ Despite this high incidence, the rate of symptomatic lymphocele is only $2.3 \% .^{3}$ Pelvic lymphocele can cause deep vein thrombosis (DVT) by venous compression. The incidence of symptomatic DVT is $0.5 \%$ in patients treated laparoscopic or robot-assisted laparoscopic radical prostatectomy for prostate cancer. ${ }^{4}$ We report our experience with pelvic lymphocele detected by symptomatic deep vein thrombosis in a patient treated with endoscopic extraperitoneal radical prostatectomy and standard pelvic lymph node dissection (PLND).

\section{Case report}

A 57-year-old man presented with lower urinary tract symptoms for 3 months. He has been on medication for his hypertension for 3 years. He was a non-smoker. The International Prostate Symptom Score was 22, maximal flow rate was $16.8 \mathrm{~mL} / \mathrm{s}$ in uroflowmetry. Serum prostate-specific antigen (PSA) and free PSA were $11.7 \mathrm{ng} / \mathrm{mL}$ and $1.1 \mathrm{ng} / \mathrm{mL}$, respectively. Prostate volume was $40 \mathrm{~mL}$ on transrectal ultrasonography. There was no palpable nodule on digital rectal examination. We performed 12-core transrectal ultrasonography-guided prostate biopsy. Adenocarcinoma was found in 4 cores of left lobe. The Gleason score was $6(3+3)$. There was low signal density on left lobe on magnetic resonance image, and no enlarged lymph node and bone metastasis. The patient was diagnosed with adenocarcinoma, clinical stage T2aNOMO.

The patient underwent endoscopic extraperitoneal radical prostatectomy with standard PLND. Our standard PLND involves the dissection and removal of lymphatic tissue from the level of the external iliac vein to the obturator nerve, extending proximally to the common iliac artery bifurcation and distal to the proximal femoral canal to include the node of Cloquet. The nodal dissection was performed by thermal energy or clipping according to the size of lymphatics. The heparin was not used for preventing DVT. Operation time was 270 minutes and it was uneventful. Prostatectomy biopsy result was prostate adenocarcinoma, Gleason score $6(3+3)$ without positive surgical margin. Pathologic stage was T2aNOM0. The number of removed pelvic lymph nodes was 7 . The JacksonPratt drain was removed on postoperative day 4. Urine leakage was detected on urethrovesical anastomosis site by urethrography postoperative day 7 . The patient was discharged with a Foley catheter on postoperative day 8 . The Foley catheter was removed on postoperative day 14 after urethrography. 
The patient came to the out-patient clinic for left-lower extremity swelling on postoperative day 20. The DVT of the femoral vein was detected on computed tomography (CT) (Fig. 1, part a). A $7 \times 3.2-\mathrm{cm}$ sized hypodense cystic structure with lobulated contours and thin regular wall was located in the parailiac region on axial CT (Fig. 1, part b). This cystic structure seemed to compress left iliac vein. There was no pulmonary thromboembolism on chest CT. Inferior vena cava filter implantation and catheter thrombolysis with urokinase $30000 \mathrm{IU} / 100 \mathrm{~mL} / \mathrm{hr}$ for catheter and heparin $100 \mathrm{IU} / \mathrm{hr}$ for sheath were performed for the treatment of DVT. Percutaneous drainage was performed for the treatment of pelvic lymphocele (Fig. 2, part a). The creatinine level of drained fluid was $0.53 \mathrm{mg} / \mathrm{dL}$. The external iliac vein stent was inserted and balloon dilatation was done 5 days later (Fig. 2, part b). The patient was discharged with a percutaneous drainage catheter on post-percutaneous drainage at 15 days. There was no fluid collection in the pelvic cavity and no thrombus iliac vein on follow-up CT 30 days later. At this time, the inferior vena cava filter and percutaneous drainage catheter were removed. At 1-year of follow-up, the patient had no symptoms of DVT and his serum PSA was at an undetectable level.

\section{Discussion}

Less than a decade ago, minimally invasive surgical techniques, such as laparoscopic prostatectomy, have evolved as an alternative to the open procedure. Despite its short history, functional and oncological outcomes have led to an increasing demand for these procedures. However, complications remain an important issue. The complication rates of retropubic radical prostatectomy and perineal prostatectomy are about $2 \%$ to $10 \%$ and $4 \%$ to $22 \%$, respectively. Complications of laparoscopic radical prostatectomy and endoscopic extraperitoneal radical prostatectomy range between $2 \%$ and $17 \% .{ }^{5}$ Rare, but possible complications, include vascular injuries, bowel injury, lymphocele formation, port-site hernia, anastomotic leakage gas embolism and catheter obstruction.

Pelvic lymphocele is a postoperative complication than can result after PLND and renal transplantation. Lymphocele forms when lymphatic channels remain after surgery. The best prevention is to avoid PLND, if possible. A PLND is not necessary in low-risk, localized prostate cancer, as the risk for positive lymph nodes does not exceed 7\%. A PLND may play a role in the treatment in all high-risk patients and in a subset of intermediate-risk patients with a $>7 \%$ nomogram predicting the risk of positive lymph nodes. ${ }^{6}$ The risk of lymphocele increases when a PLND is performed, especially if an extended template is used. ${ }^{7}$ Lymphoceles are relatively common complications, although the exact incidence is unknown since most will resolve without ever being diagnosed. They are reported to occur in $0.1 \%$ to $2 \%$ of patients, with no significant differences between surgical approaches. ${ }^{8-11}$ Meticulous cautery or clipping of lymphatic channels during open and laparoscopic prostatectomy may be important in preventing lymphoceles.

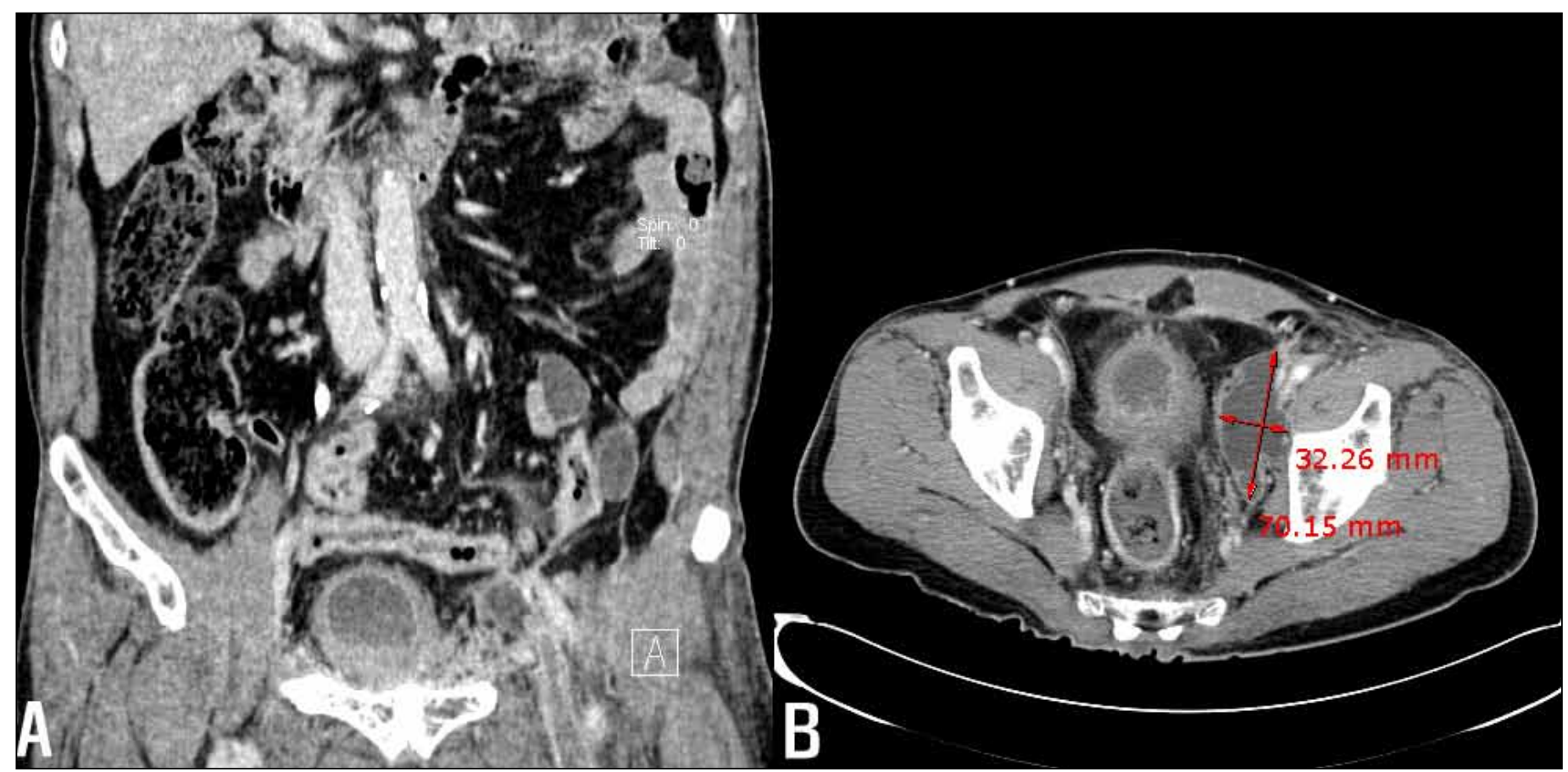

Fig. 1. Computed tomography images of deep vein thrombosis of left femoral vein (A) and $7 \times 3.2$-sized pelvic lymphocele in the left parailiac region $(B)$. 


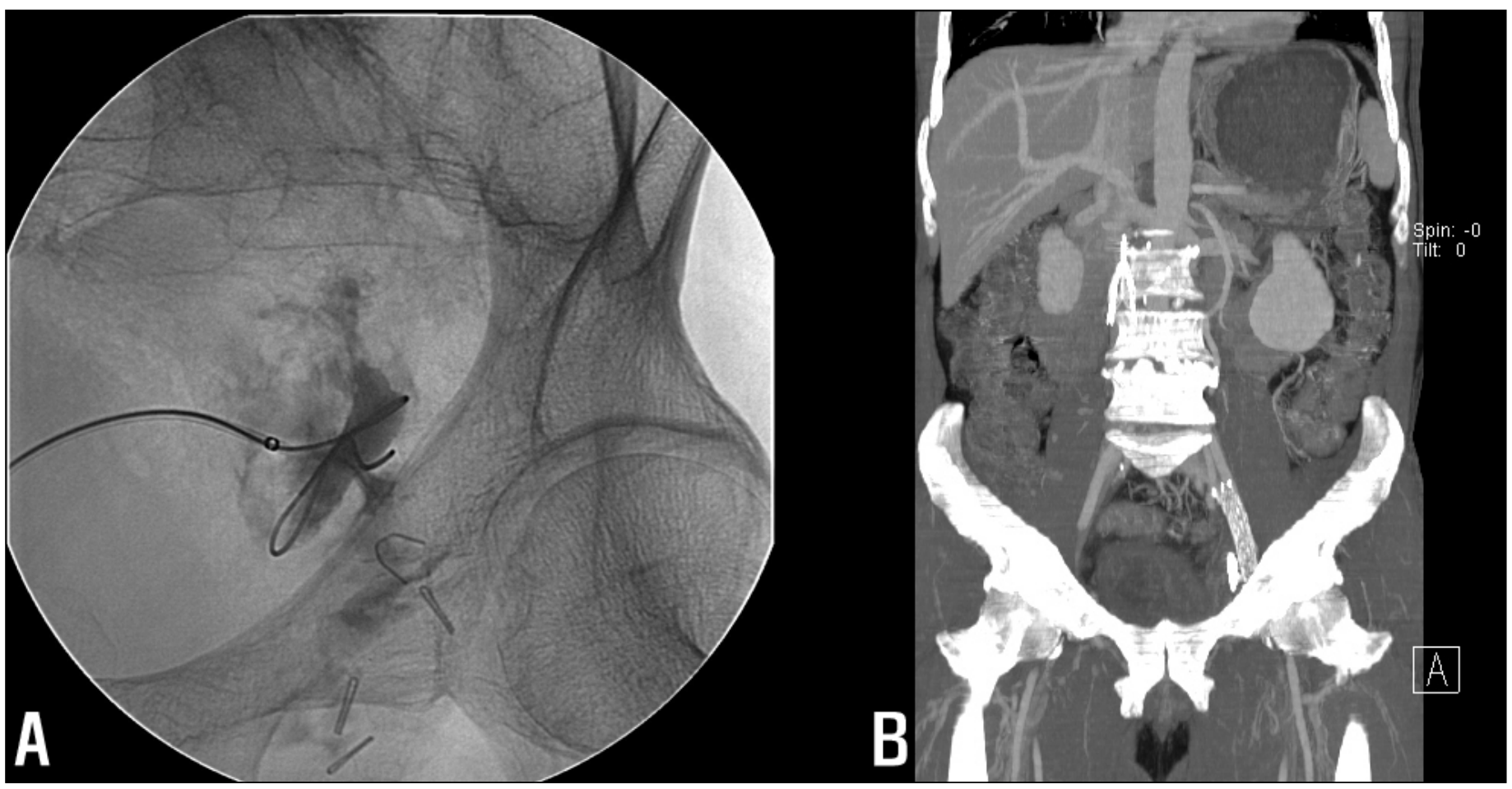

Fig. 2. Percutaneous drainage was performed for the treatment of pelvic lymphocele (A) and external iliac vein stent was inserted (B).

Many cases of Iymphocele are asymptomatic. Lymphocele-related symptoms are due to compression of adjacent structures. Lymphocele can cause hydronephrosis by compression of ureters, lower extremity edema by venous compression, constipation by rectosigmoid compression, pain by compression of pelvic nerves and urinary frequency by bladder compression. ${ }^{12}$ Fever and chills should raise the suspicion of an infected collection. Patients undergoing laparoscopic or robotic-assisted radical prostatectomy should be educated on looking for these postoperative signs to ensure a quick response and interventions thereby minimizing secondary complications.

Treatment options depend on factors, such as position, size, recurrence and infection risk. The best treatment of lymphoceles is prevention with careful lymph node dissection. Asymptomatic lymphoceles do not require treatment and disappear spontaneously. Treatment modalities included open drainage, laparoscopic drainage and percutaneous intervention. Initially, simple aspiration had been tried, but later discontinued because of high recurrence rates. Catheter drainage was used and later combined with transcatheter sclerotherapy with comparable success rates to surgical series. Numerous sclerosants have been used including povidone-iodine, ethanol, ampicillin, tetracycline, doxycyclin, bleomycin, sodium azetroate and fibrin glue. ${ }^{12}$ Marsupialization of the lymphocele via an open or laparoscopic approach should be considered when conservative therapy is unsuccessful. ${ }^{13}$ In this case, we treated lymphocele by percutaneous catheter drainage only for 31 days.

The incidence rates of DVT are about $0.2 \%$ to $0.5 \%$ in patients with prostate cancer undergoing laparoscopic or robot-assisted laparoscopic radical prostatectomy. ${ }^{4,14}$ This low incidence rates with laparoscopic series perhaps due to both faster postoperative patient mobilization and decreased transfusion rate. High-risk patients undergoing laparoscopic radical prostatectomy include prior deep vein thrombosis, current tobacco use, extended operative time and prostate size. ${ }^{3}$ According to the 2008 AUA best practice statement for the prevention of DVT, intermittent pneumatic compression devices should be used during and after all robotic-assisted laparoscopic and open procedures. Pharmacological thromboprophylaxis should be added for patients in higher-risk groups, including those with prior venous thromboembolism, hypercoagulable state or age over 60 years old. ${ }^{15}$

In our current case, the patient underwent endoscopic extraperitoneal radical prostatectomy with standard PLND for localized prostate cancer. Pelvic lymphoceles have been detected by CT during the workup of DVT. The DVT may be due to compression iliac vessel by pelvic lymphocele. Upon discharge from the hospital, all patients should be educated on the signs and symptoms of lymphocele, DVT and pulmonary embolism; they should also be advised to seek immediate care if is a concern. This is especially important since symptomatic DVT tend to occur between 1 and 3 weeks postoperatively, which is well-after most patients are discharged. Perioperative preventative measures, such 
as heparin, compressive stockings, early ambulation, using clips and limited use of PLND to indicated patients, may also help limit such complications.

Competing interests: None declared.

Acknowledgement: This paper was supported by Wonkwang University in 2010.

This paper has been peer-reviewed.

\section{References}

1. Stolzenburg JU, Rabenalt R, Do M, et al. Endoscopic extraperitoneal radical prostatectomy: the University of Leipzig experience of 2000 cases. J Endourol 2008;22:2319-25.

2. Freid RM, Siegel D, Smith AD, et al. Lymphoceles after laparoscopic pelvic node dissection. Urology 1998:51:131-4.

3. Solberg A, Angelsen A, Bergan U, et al. Frequency of lymphoceles after open and laparoscopic pelvic lymph node dissection in patients with prostate cancer. Scand I Urol Nephrol 2003;37:218-21.

4. Secin FP, Jiborn T, Biartell AS, et al. Mutti-institutional study of symptomatic deep vein thrombosis and pulmonary embolism in prostate cancer patients undergoing laparoscopic or robot-assisted laparoscopic radical prostatectomy. Eur Urol 2008:53:134-45.

5. Liatsikos $E$, Rabenalt $R$, Burchartdt $M$, et al. Prevention and management of perioperative complications in laparoscopic and endoscopic radical prostatectomy. World I Urol 2008;26:571-80.
6. Heidenreich A, Bolla M, Joniau S, et al; members of the European Association of Urology (EAU) Guidelines Office. Guidelines on Prostate Cancer. In: EAU Guidelines, edition presented at the $25^{\text {th }}$ EAU Annual Congress, Barcelona 2010.

7. Naselli A, Andreatta R, Introini C, et al. Predictors of symptomatic lymphocele after lymph node excision and radical prostatectomy. Urology 2010;75:630-5.

8. Zorn KC, Katz MH, Bernstein A, et al. Pelvic lymphadenectomy during robot-assisted radical prostatectomy: Assessing nodal yield, perioperative outcomes, and complications. Urology 2009;74:296-302.

9. Augustin $H$, Hammerer $P$, Graefen $M$, et al. Intraoperative and perioperative morbidity of contemporary radical retropubic prostatectomy in a consecutive series of 1243 patients: results of a single center between 1999 and 2002. Eur Urol 2003;43:113-8.

10. Lepor Hm Nieder AM, Ferrandino MN. Intraoperative and postoperative complications of radical retropubic prostatectomy in a consecutive series of 1000 cases. J Urol 2001;166:1729-33.

11. Treiver A, Haben B, Stark E, et al. Uni- vs. multiloculated pelvic lymphoceles: differences in the treatment of symptomatic pelvic lymphoceles after open radical retropubic prostatectomy. Int Braz I Urol 2009;35:164-9.

12. Karcaaltincaba $M, A k h a n ~ 0$. Radiologic imaging and percutaneous treatment of pelvic lymphocele. Eur J Radiol 2005:55:340-54.

13. Waples MJ, Wegenke JD, Vega RJ. Laparoscopic management of lymphocele after pelvic lymphadenectomy and radical retropubic prostatectomy. Urology 1992;39:82-4.

14. Hu JC, Nelson RA, Wilson TG, et al. Perioperative complications of laparoscopic and robotic assisted laparoscopic radical prostatectomy. J Urol 2006; 175:541-6.

15. Forrest JB, Clemens CJQ, Finamore P, et al. AUA Best Practice Statement for the prevention of deep vein thrombosis in patients undergoing urologic surgery. J Urol 2009;181:1170-7.

Correspondence: Dr. Seung Chol Park, Department of Urology, Wonkwang University School of Medicine and Hospital, 344-2 Shinyong-Dong, Iksan, Korea, 570-711, fax: +82-63-842-1455; sc.park@wonkwang.ac.kr 